\title{
Haptic Data Reduction in Multi-DoF Teleoperation Systems
}

\author{
Iason Vittorias, Hamza Ben Rached and Sandra Hirche \\ Institute of Automatic Control Engineering \\ Technische Universistät München, Germany \\ Email: \{vittorias, hirche\}@tum.de, hamza.rached@mytum.de
}

\begin{abstract}
In this paper a haptic data reduction method for multi-DoF teleoperation systems is presented. A general framework based on the deadband approach is given; it gives the flexibility, by knowledge of psychophysical properties, to form hyper-zones where information can be considered imperceivable by the human and thus does not need to be transmitted. A proper data reconstruction strategy is proposed for the receiver side to guarantee stability of global control loop despite the loss of information. Simulation and experiments on a real 7-DoF telerobotic system validate the approach and indicate high data reduction rates.
\end{abstract}

\section{INTRODUCTION}

A teleoperation system allows the human to manipulate in remote, inaccessible, dangerous, or scaled environments. Audio, vision and haptic data are exchanged between a human system interface and a teleoperator to enable the human operator to immerse into the remote environment, see Fig. 1 for a schematic overview of a teleoperation system. It enables a human to completely embody himself and feel "present" on the remote/scaled side. This can aid a range of application scenarios, from underwater or space teleoperation, teleoperation in dangerous/hazardous environments to medical applications, such as robotically mediated surgery (minimally invasive surgery, laparoscopy etc.) [1], and telementoring.

A high degree of telepresence is desired to enable operators to safely conduct teleoperation tasks. In recent years, much research has focused on the added value of haptic feedback for task performance [2], i.e. the feeling of presence in the remote side.

In teleoperation systems haptic signals are sent bidirectionally, and a global control loop is closed over the communication system. The transmission resources for typical communication networks used in teleoperation scenarios are, however, limited. Severe communication constraints are imposed by communication technology and infrastructure in space and underwater telepresence applications [3]. Generally in mobile (wireless) applications, higher network traffic is directly related to higher power consumption. High network traffic may also lead to network congestion and hence large transmission time delays and packet loss that can lead to instability of the control system or degrade performance of a force-reflecting teleoperator [4]. Data reduction methods, as well as transmission protocols, are therefore of high interest

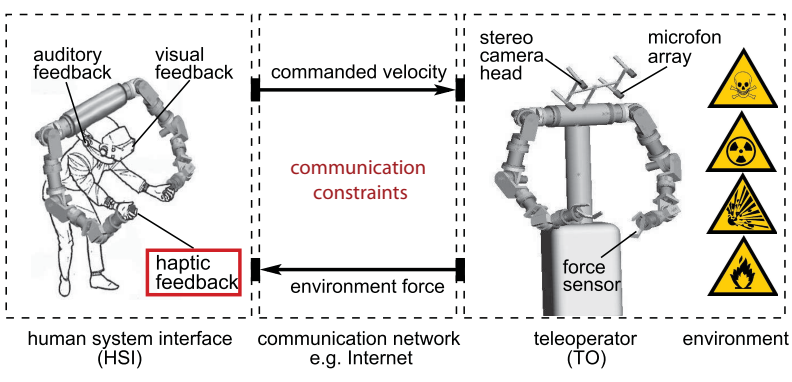

Fig. 1: Multimodal telepresence and teleaction system

for the haptic modality, just as the successfully employed standards for video (MPEG-4) and audio (MP3).

Haptic data reduction algorithms should comply with two basic teleoperation system requirements; transparency, i.e. the loss of information should be perceptually imperceivable and stability. The combined approach from a communication, control-theoretic as well as psychophysics point of view resulted in the deadband-based haptic data reduction in [5], [6]. It is shown, that the deadband-based data reduction can lead to high reduction rates. Psychophysical studies indicate that the loss of information induced by the algorithm can be considered imperceivable. Teleoperation systems with timedelay are studied in [7], and perceptual-coding in time-delayed systems is presented in [8]. Stability of the global control loop is challenging as long as information is removed from it. Therefore, all aforementioned approaches consider it and guarantee stability of the system with proper reconstruction strategies on the receiver side. However, none of those approaches is appropriate for multi degrees-of-freedom (DoF) systems. Particularly, a straigtforward extension of the developed algorithms will still lead to high data rates in multi-DoF systems.

In this work a general deadband-based framework for haptic data reduction in multi-DoF teleoperation systems is presented. Psychophysically, it is inspired by the Weber law and its multidimensional extension. It is therefore possible to integrate psychophysical findings to increase its efficiency. A proper reconstruction strategy that guarantees stability under the passivity framework is also given.

The remainder of this paper is structured as follows. In Section II the deadband-based data reduction is presented. The 
multi-DoF extension is presented in Section III. Stability issues are discussed in Section IV where the reconstruction strategy is also presented. Results are presented in Section V and the paper concludes in Section VI.

\section{Deadband-based Data Reduction for Haptic SIGNALS}

To enable telepresence and teleaction across rate-limited communication channels, e.g. in space and underwater, efficient and powerful methods to communicate and process haptic data signals are of utmost importance. A perceptionbased data reduction technique, the deadband approach, is introduced in [5], [6] and enables to reduce the packet rate within a packet-switched communication network.

The deadband approach is a lossy perceptual coding approach for haptic signals that exploits human haptic perception limits using Weber's law of Just Noticeable Differences (JND). It reduces the packet rate by removing data that is considered to be imperceivable by the human. A compression ratio of up to $90 \%$ on a 2-channel velocity-force teleoperation system is achieved without significantly impairing human immersiveness as empirically shown in human user studies [5], [6].

\section{A. Psychophysical Background}

Psychophysical studies revealed that haptic perception of force, velocity, pressure, etc. approximately follow the wellknown Weber-Fechner's law, which relates the intensity of a stimulus logarithmically to the induced sensation in the human brain [9], [10]. The psychophysical perception of a signal change is therefore proportional to the stimulus itself [11]

$$
\frac{\Delta I}{I}=\kappa,
$$

where $I$ is the stimulus and $\Delta I$ is the so called Difference Threshold or the Just Noticeable Difference (JND). It describes the smallest amount of change of stimulus $I$ which can be detected. The constant $\kappa$ describes the linear relationship between the JND and the initial stimulus $I$.

In an n-dimensional space a complicated geometry can form such deadzones where the signal is assumed to be imperceivable, e.g. Helmholts's approach, see [12]. However, the complexity of such forms makes them difficult to identify and to use. Moreover, this solution has been criticized on empirical grounds. Therefore in [12] a two-dimensional Weber law is realized by determining the stimulus coordinates for all just noticeably different stimuli in all directions. The unidimensional Weber law provides a heuristic for the formulation of a law for two or more dimensions, assuming that Weber's constants are equal along the dimensions $x$ and $y$. Therefore a JND zone can be formed, that has in general a hyperspherical form. It is considered that stimuli that lie in it, are imperceivable when the human is displayed with the initial stimulus $I$. Our work is psychophysically based on this principle.

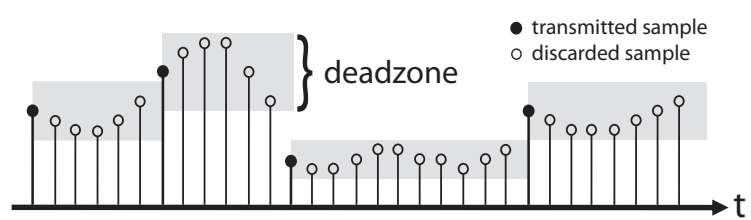

Fig. 2: Principle of the Weber-inspired deadband approach

\section{B. Deadband approach}

With the deadband approach data are sent over the communication channel only if the difference between the most recently sent sample $x\left(t^{\prime}\right)$ and the current value $x(t)$, where $t>t^{\prime}$, exceeds a threshold $\Delta_{x\left(t^{\prime}\right)}$

$$
\begin{aligned}
& \text { If: } \quad\left|x\left(t^{\prime}\right)-x(t)\right| \leq \Delta_{x\left(t^{\prime}\right)} \quad \text { Do not transmit } \\
& \text { Else: } \quad \text { Transmit new value. }
\end{aligned}
$$

Using the insights of Weber's law this threshold is chosen to grow proportionally with the magnitude of the signal $x\left(t^{\prime}\right)$

$$
\Delta_{x\left(t^{\prime}\right)}=\epsilon \cdot\left|x\left(t^{\prime}\right)\right|>0,
$$

where $\epsilon \in(0,1)$ is a factor that influences the size of the deadband. We intentionally use different notation for the deadband parameter as for the JND, i.e. $\kappa$, as the proposed compression scheme is inspired by Weber's law but not destined to identify JNDs. Therefore a meaningful choice, from the transparency point of view, would be $\epsilon \leq \kappa$. The time instant $t$ or $t^{\prime}$ will be in the following omitted for brevity; the most recently sent value $x\left(t^{\prime}\right)$ will be refered also as $\mathbf{x}^{\prime}$. If the haptic signal exceeds the perception threshold then a signal update event is triggered and the packet is transmitted. The current deadband is redefined based on the update sample's intensity value. The Weber-inspired deadband is proposed in [6] and has also been called relative deadband.

The principle of the Weber-inspired deadband approach is illustrated in Fig. 2 where only samples that exceed the deadband $\Delta$ are transmitted. Note that the deadband increases with the signal amplitude. By this means, we modify the haptic signal in a lossy but to the human user imperceptible manner. At the receiver side a decoder upsamples the signal to the local HSI and TO control sampling frequency by using a reconstruction extrapolation algorithm that will be discussed later.

\section{Multi-Dof DeAdBAnd-BAsed DatA REDUCTION}

In teleoperation systems with multi degrees-of-freedom a vector $\mathbf{x} \in \Re^{n}$ is transmitted instead of a signal. Applying the 1-DoF deadband approach to every single component of the representation is a straightforward extension, which however, turns out to be very inefficient with respect to the data transmission rate. If random movements with identically distributed directions and magnitudes of forces and velocities are examined, the component with the lowest magnitude and therefore the smallest deadband is mostly responsible for packet generation, see e.g. [6]. Moroever, the probability of 
having a component with low magnitude increases with the number of components, i.e. degrees-of-freedom, used.

The multi-DoF deadband is proposed here and aims to overcome this limitation. The approach is motivated by psychophysical results in [12] indicating the validity of the extension of Weber's Law to $n$ dimensions. Let $\mathbf{x} \in \Re^{n}$ and $\mathbf{x}^{\prime} \in \Re^{n}$ be the current and the last sent signal vectors respectively. Analog to the 1-D case, the deadband control algorithm can be defined

$$
\begin{array}{cl}
\text { If: } \quad d\left(\mathbf{x}, \mathbf{x}^{\prime}\right) \leq \Delta_{\mathbf{x}^{\prime}} & \text { Do not transmit } \\
\text { Else: } & \text { Transmit new value, }
\end{array}
$$

where $d\left(\mathbf{x}, \mathbf{x}^{\prime}\right)$ is the difference between the two stimuli $\mathbf{x}$ and $\mathrm{x}^{\prime}$ and $\Delta_{\mathbf{x}^{\prime}}$ defines the deadband zone. In this work we consider a control architecture where velocities (linear and angular) and forces/torques are transmitted between HSI and teleoperator. The velocity-force and the force-velocity architecture are typical and widely used examples for it. As a result all transmitted variables can be assumed to be from finite dimensional vector spaces. Therefore, the general case of norm, the Minkowski-norm can be used as the distance metric between the two vectors. For a given $\mathbf{x} \in \Re^{n}$, it is defined

$$
\|\mathbf{x}\|_{p}=\left(\sum_{i=1}^{n}\left|x_{i}\right|^{p}\right)^{1 / p} .
$$

In psychophysics, a special case, the Eucledian norm, i.e. $p=2$, is often used [10] and thus will be adopted here

$$
d\left(\mathbf{x}, \mathbf{x}^{\prime}\right)=\left\|\mathbf{x}-\mathbf{x}^{\prime}\right\|,
$$

where $\|$.$\| is used instead of \|.\|_{2}$ to simplify the notation. In the simplest case, the deadband $\Delta_{\mathbf{x}^{\prime}}$ can be defined analog to the $1-\mathrm{D}$ case

$$
\Delta_{\mathbf{x}^{\prime}}=\epsilon \cdot\left\|\mathbf{x}^{\prime}\right\| .
$$

The multi-DoF deadband control algorithm can thus be described as follows

$$
\begin{aligned}
& \text { If: } \quad\left\|\mathbf{x}^{\prime}-\mathbf{x}\right\| \leq \epsilon \cdot\left\|\mathbf{x}^{\prime}\right\| \quad \text { Do not transmit } \\
& \text { Else: } \quad \text { Transmit new value. }
\end{aligned}
$$

In this case the deadband zone has a hyper-spherical form; an example is illustrated in Fig. 3. However, this might not be the best choice as explained in the following. The parameter $\epsilon$ is not equal for all spatial directions; haptic perception is known to be more sensible in one direction than in an other [13]. So far the spatial distribution of the JND is not known, its empirical determination remains subject of future psychophysical studies. However, the data compression scheme in (3) can be considered conservative as the smallest JND would need to be responsible for the choice of $\epsilon$. As a result the efficiency of the data reduction mechanisc may be low for the degrees of freedom which would allow for a higher $\epsilon$.

Inspired by [12], [13], it is here proposed, to apply independent values for each corresponding dimension based on psychophysical findings instead of using the same deadband

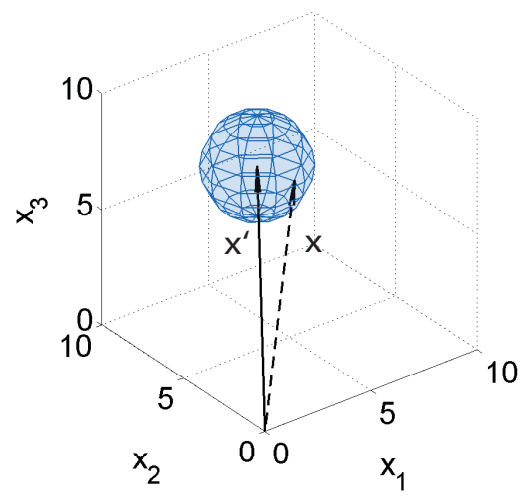

Fig. 3: Spherical 3D deadzone with $\epsilon=20 \%$. The vector with the continuous line indicates the last received vector $\mathbf{x}^{\prime}$, whereas the dashed-lined vector indicates the value $\mathbf{x}$ at the current time instance

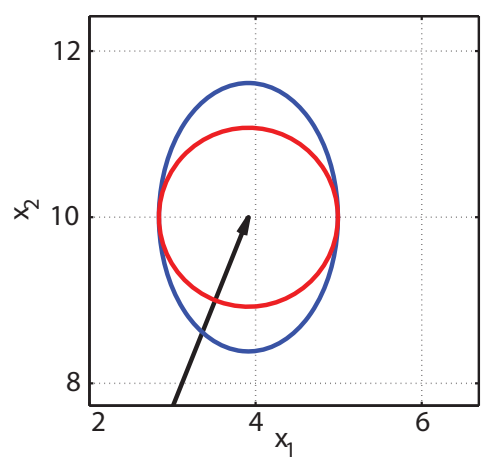

Fig. 4: 2D elliptic and spherical deadband zones for $\mathbf{x}=\left[\begin{array}{ll}4 & 10\end{array}\right], \epsilon_{x_{1}}=10 \%, \epsilon_{x_{2}}=15 \%$ and $\epsilon_{\mathbf{x}}=\epsilon_{x_{1}}=10 \%$ respectively

factor for all the vector components. This generalization of the case discussed above has the advantage of defining a larger deadband zone, that can therefore lead to further data reduction without impairing the human perception. Our proposed data reduction algorithm is now described as

$$
\left\|\Omega_{\boldsymbol{x}}\left(\mathbf{x}^{\prime}-\mathbf{x}\right)\right\| \leq\left\|\mathbf{x}^{\prime}\right\|
$$

with

$$
\boldsymbol{\Omega}_{\mathbf{x}}=\left(\begin{array}{cccc}
\frac{1}{\epsilon_{1}} & 0 & \cdots & 0 \\
0 & \frac{1}{\epsilon_{2}} & \cdots & 0 \\
\vdots & \vdots & \ddots & \vdots \\
0 & 0 & \cdots & \frac{1}{\epsilon_{n}}
\end{array}\right)
$$

a diagonal positive-definite matrix. The deadband zone is in this case an axis-aligned ellipsoid. If $\boldsymbol{\Omega}_{\mathbf{x}}$ is a non-diagonal positive-definite matrix the deadband zone is a rotated ellipsoid. The cross-terms in $\boldsymbol{\Omega}_{\mathrm{x}}$ refer here to the masking effects between the different components of the vector considered. However, as the purpose of this work is neither to study masking effects nor are there available psychophysical studies to provide values, they are set to 0 . Note that the positivedefiniteness property of $\Omega_{\mathrm{x}}$ is, here, a necessary condition. 
Since the set of changes that are unperceivable to human is bounded, the deadband zone must also form a bounded set, which is guaranteed here by $\Omega_{\mathrm{x}}$ being positive-definite. An example of an elliptical deadzone compared to the spherical is illustrated for 2-dimensions in Fig. 4.

\section{Stability Issues}

At each receiver side the local control loops still operate at the original high constant sampling rate. Accordingly, updates of the current measurement are required in each sampling instant. The fact that with the deadband algorithm fewer data packets are transmitted, requires the missing samples to be reconstructed. The data reconstruction at each receiver side has to preserve stability as this is happening inside a global closed control loop. The simplest way to reconstruct deadband signals is a zero-order-hold strategy, which however as shown in [14], does not guarantee stability. Therefore, a stabilitypreserving strategy is presented in this section. As we assume high sampling rates of the local control loops at the HSI and the teleoperator, the effects of discretization will be neglected in the following analysis: The locally controlled systems are approximated by continuous time systems.

The passivity framework, widely used in teleoperation systems, will be used here. Passivity is an energy-based concept characterizing the system by only analyzing its input/output behavior. It provides sufficient, but not necessary, input/output stability conditions. Define the power $P_{i n}$ entering a system as the scalar product between the input vector $\mathbf{u} \in \Re^{m}$ and the output vector $\mathbf{y} \in \Re^{m}$ of the system. In addition, define a lower-bounded energy storage function $S$ and a nonnegative power dissipation function $P_{\text {diss }}$, which again need not represent true physical quantities. A system is said to be passive if

$$
P_{\text {in }}=\mathbf{u}^{T} \mathbf{y}=\frac{d S}{d t}+P_{\text {diss }},
$$

i.e. if the power is either stored or dissipated in the systems. A further and important practical feature of the passivity formulation is its closure property; it implies that the combination of two passive systems connected in either a feedback or parallel configuration is again passive [15].

For the network model shown in Fig. 5 the passivity condition can be formulated as follows

$$
\int_{0}^{t}(\underbrace{\mathbf{u}^{T} \cdot \mathbf{y}^{*}}_{P_{M}}-\underbrace{\mathbf{y}^{T} \cdot \mathbf{u}^{*}}_{P_{S}}) d \tau \geq 0 \quad \forall t>0,
$$

where the $*$ indicates reconstructed values, $P_{M}$ represents the power on the master side, and $P_{S}$ the power on the slave side. Unfortunately, the term $P_{M}$ is not known on the slave side, and the term $P_{S}$ is not known on the master side. A direct comparison between those two terms is thus not possible for passivity check. It is therefore here proposed to maximize $\mathbf{u}^{T} \cdot \mathbf{y}^{*}$ and minimize $\mathbf{u}^{* T} \cdot \mathbf{y}$ to guarantee there exist no vector $\mathbf{y}$ that generates more energy then the $\mathbf{y}^{*}$ we are using for reconstruction, and no vector $\mathbf{u}$ that generates less energy

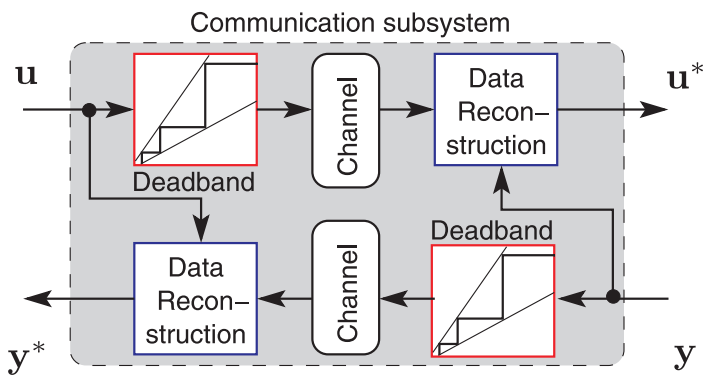

Fig. 5: A 2-port model of the network with deadband control

then $\mathbf{u}^{*}$. This is a conservative reconstruction strategy which guarantees passivity.

The problem is formulated as a constrained optimization problem where the reconstructed value $\mathbf{u}^{*}$ and $\mathbf{y}^{*}$ will guarantee that the minimum and maximum energy is generated, respectively. For brevity, here, we will show the minimization problem and reconstruction strategy for the forward channel, i.e. the reconstruction vector $\mathbf{u}^{*}$. The proof is similar for the reconstruction vector $\mathbf{y}^{*}$.

The reconstruction vector $\mathbf{u}^{*}$ should at each time instance minimize the power under the constraints a) that it lies in the deadband zone and b) that the power is positive. The first constraint ensures the transparency of the reconstruction algorithm, and the second is defined to guarantee that the reconstruction algorithm will not change the power flow direction. The minimization problem is

$$
\begin{array}{rll}
\text { Minimize } & p\left(\mathbf{u}^{*}\right) & =\mathbf{u}^{*} \cdot \mathbf{y} \\
\text { with } & c_{1}\left(\mathbf{u}^{*}\right) & =\left\|\boldsymbol{\Omega}_{\mathbf{u}^{*}}\left(\mathbf{u}^{\prime}-\mathbf{u}^{*}\right)\right\| \leq\left\|\mathbf{u}^{\prime}\right\| \\
\text { and } & c_{2}\left(\mathbf{u}^{*}\right) & =\mathbf{u}^{*} \cdot \mathbf{y} \geq 0
\end{array}
$$

and can be solved using the Karush-Kuhn-Tucker method [16] by solving the following conditions

$$
\begin{array}{lll}
\mathbf{u}^{*} \mathcal{L}_{\mathbf{u}^{*}} & = & 0 \\
\lambda_{1} \mathcal{L}_{\lambda_{1}} & = & 0 \\
\lambda_{2} \mathcal{L}_{\lambda_{2}} & = & 0 \\
\lambda_{1} \geq 0 & \text { and } & c_{1}\left(\mathbf{u}^{*}\right) \leq\left\|\mathbf{u}^{\prime}\right\| \\
\lambda_{2} \geq 0 & \text { and } & c_{2}\left(\mathbf{u}^{*}\right) \geq 0,
\end{array}
$$

where

$$
\mathcal{L}\left(\mathbf{u}^{*}, \lambda_{1}, \lambda_{2}\right)=p\left(\mathbf{u}^{*}\right)+\lambda_{1}\left(c_{1}\left(\mathbf{u}^{*}\right)-\left\|\mathbf{u}^{\prime}\right\|\right)-\lambda_{2} c_{2}\left(\mathbf{u}^{*}\right) .
$$

and $\mathcal{L}_{\mathbf{u}^{*}}, \mathcal{L}_{\lambda_{1}}, \mathcal{L}_{\lambda_{2}}$ the partial derivative of $\mathcal{L}\left(\mathbf{u}^{*}, \lambda_{1}, \lambda_{2}\right)$ with respect to $\mathbf{u}^{*}, \lambda_{1}$ and $\lambda_{2}$, respectively. A solution exists when $p\left(\mathbf{u}^{*}\right), c_{1}\left(\mathbf{u}^{*}\right)$ and $c_{2}\left(\mathbf{u}^{*}\right)$ are real-valued differentiable functions.

Solving the optimization problem yields

$$
\mathbf{u}^{*}=\mathbf{u}^{\prime}-\frac{\boldsymbol{\Omega}_{\mathbf{u}}^{-1} \mathbf{\Omega}_{\mathbf{u}}^{-T} \mathbf{y}}{\left\|\boldsymbol{\Omega}_{\mathbf{u}}^{-T} \mathbf{y}\right\|}\left\|\mathbf{u}^{\prime}\right\| .
$$

Remark 1: In the case of spherical deadband, (6) can be simplified to

$$
\mathbf{u}^{*}=\mathbf{u}^{\prime}-\frac{\mathbf{y}}{\|\mathbf{y}\|} \epsilon_{\mathbf{u}}\left\|\mathbf{u}^{\prime}\right\| .
$$


Similarly to the expression for $\mathbf{u}^{*}$ in (6) a maximization problem can be solved for $\mathbf{u}^{\mathbf{T}} \mathbf{y}^{*}$, which is equivalent to mimization of $-\mathbf{u}^{\mathbf{T}} \mathbf{y}^{*}$ to compute the proper reconstruction strategy for $\mathbf{y}^{*}$. Using the same constrained optimization method

$$
\mathbf{y}^{*}=\mathbf{y}^{\prime}+\frac{\boldsymbol{\Omega}_{\mathbf{y}}^{-1} \boldsymbol{\Omega}_{\mathbf{y}}^{-T} \mathbf{u}}{\left\|\boldsymbol{\Omega}_{\mathbf{y}}^{-T} \mathbf{u}\right\|}\left\|\mathbf{y}^{\prime}\right\|,
$$

which again for the spherical deadband can be simplified to

$$
\mathbf{y}^{*}=\mathbf{y}^{\prime}+\frac{\mathbf{u}}{\|\mathbf{u}\|} \epsilon_{\mathbf{y}}\left\|\mathbf{y}^{\prime}\right\| .
$$

Remark 2: The above results comply in the 1-DoF case with the result in [5], [7].

\section{A. Position Drift}

The deadband control for the velocity signal induces a velocity error between the HSI and the teleoperator. Velocitybased architectures generally lead to position and orientation drift in the systems due to the integration error of the controllers. As a result the teleoperator position drifts away from the HSI position. The position drift does not only deteriorate the transparency, but may also drive the system to inoperability if the HSI or the teleoperator reaches the limit of its workspace. In [17] a time-delayed velocity/force architecture is extended by a position feedforward. It is designed with a saturated position controller at the teleoperator such that the passivity condition is not violated. Here, a pose update strategy with a closed-loop kinematic control in joint space, as in [18], is used instead. A pose update, i.e. position and orientation, is transmitted together with the velocity data packets to improve the position tracking. This does not create any considerable load on the network, since the packet payload is small compared to the header of an IP-packet, and no new packet is transmitted.

The orientation and position errors introduced by the deadband control are considered as kinematic disturbances. The updated pose values are used in the closed kinematic controller in the sample time when they are received. For the rest of the time the kinematic control loop is considered open until a new update arrives. The closed-loop kinematic equation has the following form

$$
\dot{\boldsymbol{\theta}}=\mathbf{J}^{-1}\left[\begin{array}{c}
\mathbf{v}^{*}+\mathbf{K}_{t} \mathbf{e}_{t} \\
\boldsymbol{\omega}^{*}+\mathbf{K}_{o} \mathbf{e}_{o}
\end{array}\right]
$$

where $\mathbf{v}^{*}$ and $\boldsymbol{\omega}^{*}$ the reconstructed linear and angular target velocities, $\theta$ the joint angles, $\mathbf{J}$ the Jacobian matrix of the robot, $\mathbf{K}_{t}$ and $\mathbf{K}_{o}$ the kinematic controller gains, and $\mathbf{e}_{t}$, $\mathbf{e}_{o}$ the translational and orientational error. Asymptotic stability of this kinematic control is proven in [18].

In some telerobotic scenarios, a maximum position drift $\tilde{x}_{\max }$ is allowed. There exists, therefore, a maximal period of time $T_{\max }$ that can elapse without new packets being sent in case a movement in constant velocity for example is happening. By knowledge of the maximum error, based on the last velocity value sent, and on the deadband parameter $\epsilon_{\max }$

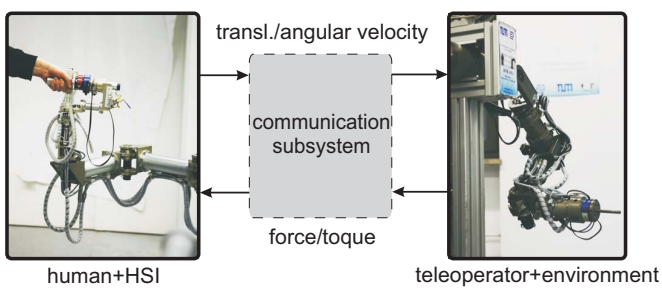

Fig. 6: The 7-DoF teleoperation setup used for the experiment. The communication subsystem is visualized in Fig. 5

we can compute the maximum period $T_{\max }$ so that the upper bound of the position drift is not violated

$$
\begin{aligned}
2 \epsilon_{\max }\left\|\mathbf{v}^{\prime}\right\| T_{\max } & =\tilde{x}_{\max } \\
\Rightarrow T_{\max } & =\frac{\tilde{x}_{\max }}{2 \epsilon_{\max }\left\|\mathbf{v}^{\prime}\right\|} .
\end{aligned}
$$

Therefore, an alternative position update strategy can be used. Position updates are transmitted together with the velocity updates. If, however, the maximum allowable time $T_{\max }$ is exceeded between two consecutive transmission, a position update packet is triggered to avoid exceeding the maximum allowable drift.

\section{Evaluation}

The multi-DoF deadband approach is tested on a teleoperation system consisting of a master 7-DoF manipulator, and a human-scaled 7-DoF robotic arm for the teleoperator. Each robot has a 6-DoF force/torque sensor mounted on its endeffector. Both robots are admittance-type devices and are thus controlled using a position-based admittance control scheme. Gravity and external forces are compensated. The wrench and twist of each endeffector are transmitted through the channel, i.e a 6-dimensional vector with tranlational and angular velocity on the forward channel, and a 6-dimensional vector with cartesian forces and torques. The environment consists of free space and a silicon cube with stiffness $1400 \mathrm{~N} / \mathrm{m}$. The network channel consists of simple LAN with $100 \mathrm{MB} / \mathrm{s}$ bandwidth. Time delay is therefore considered negligible. Deadband is applied on both channels and the reconstruction strategy given in (6) and (7) is applied at each receiver side.

The experiment consists of a free space motion and a contact phase where a silicon cube is haptically explored. The contact occured in a constant angle near $45^{\circ}$, so both the $\mathrm{x}$ - and zcomponents of the force were triggered. During the interaction the deadband algorithm switched between the multi-DoF deadband approach and the straightforward extension of the 1-DoF deadband seen in the beginning of Section III; from now on will be mentioned as component-wise deadband. The same $\epsilon$ is used in each dimension for simplicity. The displayed stiffness to the human is then estimated using least-squares identification.

Results are illustrated in Fig. 7. It is observed that the multi-DoF deadband can lead to high data reduction without significant error on the stiffness, i.e. for $90 \%$ of packets reduced the stiffness error was measured to be below $5 \%$. 


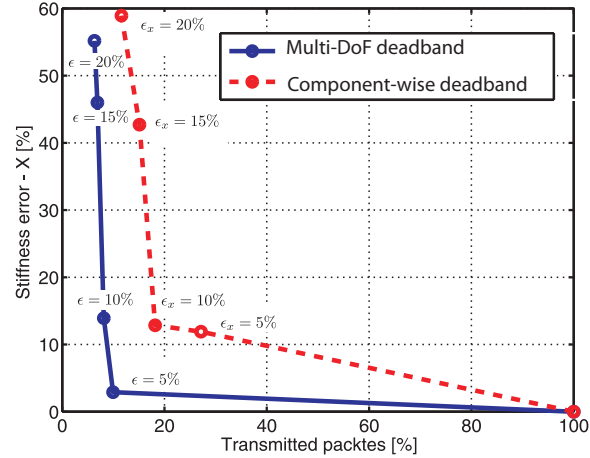

(a)

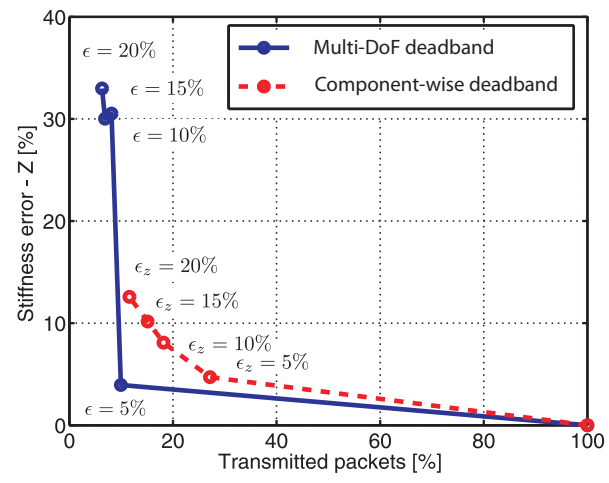

(b)

Fig. 7: Stiffness error : a) X-component, b) z-component

Psychophysical studies indicate a stiffness error up to $8 \%$ to be imperceivable for pinch/finger movements [19] whereas the stiffness error perception threshold is increased up to $23 \%$ for the arm/forearm as shown in [20], [21]. Moreover, the fact that the curve for the multi-DoF deadband lies always below the component-wise deadband in both figures indicates the increased performance of the proposed approach, namely for the same amount of transmitted packets less stiffness error is measured.

\section{CONCLUSION}

In this paper a haptic data reduction technique for multiDoF systems is presented based on previous results that use deadband control. The method proposed enables psychophysical findings on the human haptic perception to be easily integrated for each DoF. A reconstruction strategy is also given for each receiver side, to guarantee stability of the global haptic control loop closing over the communication channel. Experimental results on a 7-DoF teleoperation setup show the improved performance of the proposed approach in transmitted packet as well as in stiffness error. Future work will include psychophysical studies.

\section{ACKNOWLEDGMENT}

Authors would like to thank Prof. Eckehard Steinbach and Julius Kammerl for their constructive comments. This work is supported by the German Research Foundation (DFG) within the Collaborative Research Center SFB453 on "High-Fidelity Telepresence and Teleaction".

\section{REFERENCES}

[1] J. Rosen and B. Hannaford, "Doc at a distance," Spectrum, IEEE, vol. 43, no. 10 , pp. 34-39, Oct. 2006.

[2] J. Dennerlein, D. Martin, and C. Hasser, "Force-feedback improves performance for steering and combined steering-targeting tasks," Proceedings of the SIGCHI conference on Human factors in computing systems, The Hague, The Netherlands, pp. 423-429, 2000.

[3] I. F. Akyildiz, D. Pompili, and T. Melodia, "Challenges for efficient communication in underwater acoustic sensor networks," SIGBED Rev., vol. 1, no. 2, pp. 3-8, 2004.

[4] J. Vertut, A. Micaelli, P. Marchal, and J. Guittet, "Short transmission delay on a force reflective bilateral manipulator," in Proc. 4th RomanSymposium, Poland, 1981.

[5] S. Hirche, P. Hinterseer, E. Steinbach, and M. Buss, "Transparent data reduction in networked telepresence and teleaction systems. part i: Communication without time delay," Presence: Teleoperators \& Virtual Environments, vol. 16, no. 5, pp. 523-531, 2007. [Online]. Available: http://www.mitpressjournals.org/doi/abs/10.1162/pres.16.5.523

[6] P. Hinterseer, S. Hirche, S. Chaudhuri, E. Steinbach, and M. Buss, "Perception-based data reduction and transmission of haptic data in telepresence and teleaction systems," Signal Processing, IEEE Transactions on, vol. 56, no. 2, pp. 588-597, Feb. 2008.

[7] S. Hirche and M. Buss, "Transparent data reduction in networked telepresence and teleaction systems. part ii: Time-delayed communication," Presence: Teleoperators \& Virtual Environments, vol. 16, no. 5, pp. 532-542, 2007. [Online]. Available: http://www.mitpressjournals.org/doi/abs/10.1162/pres.16.5.532

[8] I. Vittorias, J. Kammerl, S. Hirche, and E. Steinbach, "Perceptual coding of haptic data in time-delayed teleoperation," in EuroHaptics conference, 2009 and Symposium on Haptic Interfaces for Virtual Environment and Teleoperator Systems. World Haptics 2009. Third Joint, March 2009, pp. 208-213.

[9] G. C. Burdea, Force and Touch Feedback for Virtual Reality. Wiley, 1996.

[10] G. A. Gescheider, Psychophysics. Lawrence Erlbaum, 1985.

[11] E. H. Weber, Die Lehre vom Tastsinn und Gemeingefuehl, auf Versuche gegruendet. Vieweg: Braunschweig, Germany, 1851.

[12] J. Droesler, "An n-dimensional weber law and the corresponding fechner law," Journal of Mathematical Psychology, vol. 44, pp. 330-335, 2000.

[13] H. Pongrac, P. Hinterseer, J. Kammerl, E. Steinbach, B. Faerber, U. D. B. Muenchen, and T. U. Muenchen, "Limitations of human 3d-force discrimination," 2006. [Online]. Available: http: //citeseerx.ist.psu.edu/viewdoc/summary?doi=10.1.1.68.8597

[14] R. Adams and B. Hannaford, "Stable haptic interaction with virtual environments," IEEE Transactions on Robotics and Automation, vol. 15, no. 3, pp. 465-474, 1999.

[15] K. P. Sepulchre R., Jankovic M., Constructive Nonlinear Control, 1st ed. Springer-Verlag, 1997.

[16] S. K. Mishra, S. Z. Wang, and K. K. Lai, Generalized Convexity and Vector Optimization. Springer Verlag, 2009.

[17] N. Chopra, M. Spong, S. Hirche, and M. Buss, "Bilateral teleoperation over the internet: the time varying delay problem," in American Control Conference, 2003. Proceedings of the 2003, vol. 1, 4-6 June 2003, pp. 155-160.

[18] R. Campa, R. Kelly, and E. Garcia, "On stability of the resolved acceleration control," vol. 4, pp. 3523 - 3528 vol.4, 2001.

[19] H. Tan, N. Durlach, G. Beauregard, and M. Srinivasan, "Manual discrimination of compliance using active pinch grasp: The role of force and work cues," Perception and Psychophysics, vol. 57, pp. 495-510, 1995.

[20] L. A. Jones and I. W. Hunter, "Human operator perception of mechanical variables and their effects on tracking performance," ASME Advances in Robotics, vol. 53, pp. 42-49, 1992.

[21] —_, "A perceptual analysis of stiffness," Experimental Brain Research, vol. 79, pp. 150-156, 1990. 